PACS 71.18.+y, 71.20.-b

\title{
Splitting the eigenvectors space for Kildal's Hamiltonian
}

\author{
G.P. Chuiko ${ }^{1}$, N.L. Don ${ }^{2}$ \\ ${ }^{1}$ Petro Mohyla Black Sea State University, Department of Medical Devices and Systems, \\ 10, 68 Desantnikov str., 54003 Mykolaiv, Ukraine; e-mail: gp47@mail.ru \\ ${ }^{2}$ Kherson National Technical University, Department of General and Applied Physics, \\ 24, Beryslawskoe Shosse, 73008 Kherson, Ukraine; e-mail: n_don@mail.ru
}

\begin{abstract}
The rational canonical form of Kildal's Hamiltonian has been obtained as a matrix with two identical diagonal blocks. It allowed to formulate and strictly prove few common assertions. Each of the eigenvalues of Kildal's Hamiltonian is twice degenerated everywhere, and it is well-known Kramers' degeneration, firstly. However, there is neither degeneration with except for Kramers', secondly. The symmetry of Kildal's Hamiltonian forcedly includes the operation of inversion (i.e. the center of symmetry), thirdly. Consequently this form of Hamiltonian is evidently not able to describe the specific properties of crystals without the center of symmetry. The Frobenius form (alias "the rational canonical form") of Hamiltonian should consist of two non-identical diagonal blocks to remove Kramers' degeneration.
\end{abstract}

Keywords: Kildal's Hamiltonian, Kramers' degeneration, splitting the space of eigenvectors, rational canonical form.

Manuscript received 08.12.10; accepted for publication 02.12.10; published online 30.12.10.

\section{Introduction}

The problem of the splitting the eigenvector space is solved here for Hamiltonians of Kildal's type [1]. Mathematical and physical conclusions following from the analysis both of rational canonical form of Kildal's Hamiltonian as well as of characteristic polynomials associated with it will be proved and presented. We are going to get the strong evidences of everywhere presented Kramer's degeneration of each energy level for this kind of Hamiltonian. Conversely, there is no other degeneration except for that as follows.

The original Hamiltonian of Kildal was written down with such a basis [1]:

$V_{b}=\left\langle i S_{\beta}, m(X-i Y)_{\alpha}, Z_{\beta}, m(X+i Y)_{\alpha}\right.$,

$\left.i S_{\alpha},-m(X+i Y)_{\beta}, Z_{\alpha}, m(X-i Y)_{\beta}\right\rangle$,

where $S, X, Y, Z$ are basis functions of the corresponding irreducible representations (scalar and vector): $\alpha, \beta$ are two spin indicators whereas $m=\frac{1}{\sqrt{2}}$ and $i^{2}=-1$.

The large-blocked structure of this Hamiltonian has the form
$\mathbf{H}=\left(\begin{array}{cc}\mathbf{H}_{1} & \mathbf{H}_{2} \\ \mathbf{H}_{2}^{\oplus} & \mathbf{H}_{1}\end{array}\right)$,

where $\mathbf{H}_{2}^{\oplus}$ is submatrix Hermitian transpose to $\mathbf{H}_{2}$ and submatrices $\mathbf{H}_{1}$ and $\mathbf{H}_{2}$ have the following forms:

$$
H_{1}=\left(\begin{array}{cccc}
E_{s} & 0 & k_{z} P & 0 \\
0 & E_{p}+(\delta-\Delta) / 3 & \Delta \sqrt{2} / 3 & 0 \\
k_{z} P & \Delta \sqrt{2} / 3 & E_{p}-2 \delta / 3 & 0 \\
0 & 0 & 0 & E_{p}+(\delta+\Delta) / 3
\end{array}\right),
$$

$H_{2}=\left(\begin{array}{cccc}0 & -m P\left(k_{x}+i k_{y}\right) & 0 & m P\left(k_{x}-i k_{y}\right) \\ m P\left(k_{x}+i k_{y}\right) & 0 & 0 & 0 \\ 0 & 0 & 0 & 0 \\ m P\left(k_{x}-i k_{y}\right) & 0 & 0 & 0\end{array}\right)$,

where the symbols $E_{s}, E_{p}, \Delta, \delta, P$ mean the same as in [1]: energies of $s$ - and $p$-states, parameter of spin splitting, Kildal's parameter of crystalline field and matrix element of the quasi-pulse. Symbols $k_{x}, k_{y}, k_{z}$ denote three components of the wave vector. 
We would like to transform the Hamiltonian (2) with blocks (3) and (4) to the block-diagonal form to split its eigenvectors space by a couple of independent and orthogonal subspaces. This will be helpful to formulate several strong and quite general statements about eigenvectors and eigenvalues of such a kind of Hamiltonians.

\section{Solving the problem}

Let us denote:

$E_{g}=E_{s}-E_{p}-\frac{(\delta-\Delta)}{3}$.

Let us use thereto the spherical coordinates, where

$k_{x} \pm i k_{y}=k \sin (\theta) e^{ \pm i \varphi}$

and $k=\sqrt{k_{x}^{2}+k_{y}^{2}+k_{z}^{2}}$.

The submatrices (3) and (4) shall get the following forms after these substitutions and the relocate of the energy zero-point $\left(E_{p}+\frac{\delta+\Delta}{3}=0\right)$ :

$$
\begin{aligned}
H_{1} & =\left(\begin{array}{cccc}
E_{g} & 0 & k P \cos (\tilde{\Gamma}) & 0 \\
0 & -2 \Delta / 3 & \Delta \sqrt{2} / 3 & 0 \\
k P \cos (\theta) & \Delta \sqrt{2} / 3 & -\Delta-\delta / 3 & 0 \\
0 & 0 & 0 & 0
\end{array}\right), \\
H_{2} & =m k P \sin (\theta)\left(\begin{array}{cccc}
0 & -e^{i \varphi} & 0 & e^{-i \varphi} \\
e^{i \varphi} & 0 & 0 & 0 \\
0 & 0 & 0 & 0 \\
e^{-i \varphi} & 0 & 0 & 0
\end{array}\right) .
\end{aligned}
$$

The rational canonical form (or the so-called "Frobenius' form") of this Hamiltonian can be found using such a system of computer mathematics as Maple11, where this possibility is a part of the package of programmes called "Linear Algebra". It is possible to obtain not only the partly diagonalized Frobenius' form of Hamiltonian, but the matrix of the basis transformation $(Q)$ also by this way. However, this matrix is very bulky and therefore is absent here in paper.

The rational canonical form of Kildal's Hamiltonian may be written down in a kind:

$$
\mathbf{H}_{F r}=\left(\begin{array}{cc}
\mathbf{H}_{\mathbf{1}} & \mathbf{0} \\
\mathbf{0} & \mathbf{H}_{\mathbf{1}}
\end{array}\right) .
$$

Thus, the Frobenius form of Hamiltonian has the block-diagonal kind with identical $(4 \times 4)$ submatrices on the main diagonal:

$$
H_{1}=\left(\begin{array}{cccc}
0 & 0 & 0 & \delta \Delta(k P \sin (\theta))^{2} / 3 \\
1 & 0 & 0 & 2 \delta \Delta E_{g}+k^{2} P^{2}\left(\delta \sin (\theta)^{2}+2 \Delta / 3\right) \\
0 & 1 & 0 & E_{g}(\delta+\Delta)-2 \Delta / 3+k^{2} P^{2} \\
0 & 0 & 1 & E_{g}-\delta-\Delta
\end{array}\right) .
$$

Now we can get the dispersion law as the submatrix (10) characteristic polynomial with its dependence on energy $(E)$ :

$$
\begin{aligned}
& E^{4}-\left(E_{g}-\delta-\Delta\right) E^{3}-\left(E_{g}(\delta+\Delta)-2 \Delta / 3+k^{2} P^{2}\right) E^{2}- \\
& -\left(2 \delta \Delta E_{g}+k^{2} P^{2}\left(\delta \sin (\theta)^{2}+2 \Delta / 3\right)\right) E- \\
& -\delta \Delta(k P \sin (\theta))^{2} / 3=0 .
\end{aligned}
$$

It is worthy to note that coefficients of the dispersion law (11) are presented in the latter column of (10) as elements of this matrix. This equation may be resolved directly and even in radicals because it is algebraic equation of the fourth degree. However, its four roots are somewhat bulky and thus are inconvenient for analysis. The indirect solutions (anything like $k(E, \theta)$ or even $\left.k^{2}(E, \theta)\right)$ are looking much better from this point of view. By the way, using these indirect solutions is old good tradition still from Kane [2]. So, we have from (11):

$k^{2}(E, \theta)=\frac{E\left(E_{g}-E\right)\left((3 E+2 \Delta) \delta+3 \Delta E+3 E^{2}\right)}{P^{2}\left((3 E+\Delta) \delta \sin (\theta)^{2}+2 \Delta E+3 E^{2}\right)}$.

Our formula for the dispersion law is slightly more compact in comparison with the original one [1]:

$$
\begin{gathered}
k^{2}(E, \theta)=\frac{E\left(E_{g}-E\right)\left((E+2 \Delta / 3)(E+\delta+\Delta / 3)-2 \Delta^{2} / 9\right)}{P^{2}\left[\left((E+\Delta / 3)(E+\delta+\Delta / 3)-\Delta^{2} / 9\right) \sin (\theta)^{2}+\right.} . \\
\left.+E(E+2 \Delta / 3) \cos (\theta)^{2}\right]
\end{gathered}
$$

Nevertheless, if we will find a difference between both expressions, we shall get zero. What means that expressions (12) and (13) are equivalent and tantamount.

\section{Conclusions}

\section{A. Mathematical point of view}

The block-diagonal structure of the Frobenius matrix (9) determines that [3-5]:

1. Characteristic polynomials for both identical diagonal blocks (of submatrices) are also identical.

2. At the same time, the characteristic polynomials are the minimum polynomials of diagonal submatrices (i.e. of blocks).

3. The sequence $(1,1,1, \operatorname{Pol}(E))$ where $\operatorname{Pol}(E)$ is the characteristic polynomial of the submatrix $H_{1}$ (left side of Eq. (11)) is the sequence of all invariant polynomials of the submatrix $E I-H_{1}$, where $I$ is the identity matrix and $H_{1}$ is the submatrix determined by (10).

4. The characteristic polynomial of the Hamiltonian matrix $\mathbf{H}$ is a product of all its invariant polynomials. Consequently, each of two identical 
invariant polynomials $\operatorname{Pol}(E)$ divides the characteristic polynomial of the matrix $\mathbf{H}$ without a rest.

5. The identity of characteristic and minimum polynomials is the guaranty that all eigenvalues of the submatrix $H_{1}$ are different in pairs.

6. If $E_{j}$ is one of four $(j=1,2,3,4)$ roots of the submatrix characteristic polynomial $(\operatorname{Pol}(E))$, then one and only one eigenvector corresponds to this root, and this eigenvector has the following coordinates with the basis functions of Frobenius' form (9): $\left\langle 1, E_{j}, E_{j}^{2}, E_{j}^{3}\right\rangle$.

7. Eigenvectors mentioned above belongs to one of these two orthogonal 4D-subspaces with the basis functions of Frobenius' form (9), and the complete 8Dspace of eigenvectors is the direct sum of both subspaces.

\section{B. Physical point of view}

1. The points $1,3,4$ and 7 of the above list mean that each of eigenvalues of Kildal's Hamiltonian is twice degenerated, and it is well-known Kramers' degeneration evidently.

2. The points 2,5 and 6 testify that there is neither degeneration with except for Kramers'.

3. The symmetry of Kildal's Hamiltonian forcedly includes the operation of inversion (i.e. the center of symmetry).
Consequently this form of Hamiltonian is evidently not able to describe the specific properties of crystals without the center of symmetry. To remove Kramers' degeneration, the Frobenius form of Hamiltonian should consist of two non-identical diagonal blocks, as it has been explained in [6]. By the way, basis functions of more general Hamiltonian [6] are "one to one" as those for Hamiltonian (9) and can be found with the matrix $\mathbf{Q}$ mentioned above and got by us.

\section{References}

1. H. Kildal, Band structure of $\mathrm{CdGeAs}_{2}$ near $\mathrm{k}=0$ // Phys. Rev. 10(12), p. 5082-5087 (1974).

2. E.O. Kane, Band structure of indium antimonide // J. Phys. Chem. Solids 1, p. 249-261 (1957).

3. V.V. Voievodin, Yu.A. Kuznetsov, Matrices and Calculations. Nauka, Moscow, 1984 (in Russian).

4. F.R. Gantmacher, The Theory of Matrices. Nauka, Moscow, 1967 (in Russian).

5. K.G. Valeiev, Splitting the Matrix Spectrum. Vyshcha shkola, Kiev, 1986 (in Russian).

6. G.P. Chuiko, V.V. Martyniuk and V.K. Bazhenov, The basic peculiarities of the band spectra within the generalized model by Kildal for one-axis semiconductors // Semiconductor Physics, Quantum Electronics \& Optoelectronics, 8(2), p. 28-31 (2005). 